phys. stat. sol. (b) 204, 283 (1997)

Subject classification: 73.61.Ey; 78.66.Fd; S7.12; S13

\title{
Energy Relaxation of Photo-Excited Hot Electrons under an External Electric Field in a Quasi-One-Dimensional Structure
}

\author{
S. Niwa ${ }^{1}$ ), M. Yamaguchi, N. Sawaki, and T. Suzuki ${ }^{2}$ ) \\ Department of Electronics, Nagoya University, Chikusa-ku, Nagoya 464-01, Japan \\ Tel.: +81-52-789-3321; Fax: +81-52-789-3157; e-mail: sawaki@nuee.nagoya-u.ac.jp
}

(Received August 1, 1997)

\begin{abstract}
Using the photoluminescence intensity correlation method, the transient distribution of photo-excited hot electrons under an external electric field is studied in a quasi-one-dimensional structure to reveal the competition of the heating effect and the cooling effect due to emission of LA and LO phonons. Temporal accumulation of carriers around the LO phonon emission threshold was achieved by heating the carriers with the external electric field.
\end{abstract}

Introduction. The size quantization or the mesoscopic effect modifies the electronphonon interaction in small devices, resulting in novel transport as well as the optical properties at short time scales. In this paper, we will study the transient distribution of photo-excited hot electrons in a quasi-one-dimensional (Q1D) structure with the aid of the femtosecond photoluminescence (PL) intensity correlation method [1]. Since the signal is due to the cross correlation of the photo-excited electron and hole densities, we may characterize the photo-excited non-equilibrium electrons in n-type doped quantum wells.

Sample Preparation and Experimental Method. A $14 \mathrm{~nm}$ GaAs quantum well (QW) embedded between AlGaAs cladding layers was grown by MBE on a semi-insulating GaAs substrate. The Q1D structure was made using the laser holography and wet chemical etching, of which geometrical width was about $300 \mathrm{~nm}$ [2]. Ohmic contacts were provided at both ends of wires so that we might apply an electric field along the wire axis. The S-dH measurements showed that a wire has carriers at $2.5 \times 10^{6} \mathrm{~cm}^{-1}$ with the effective width of $133 \mathrm{~nm}$ [2]. The femtosecond PL intensity correlation signal was measured using a CPM dye laser (pulse width $150 \mathrm{fs}$, interval $10 \mathrm{~ns}$, wavelength $610 \mathrm{~nm})$ at $77 \mathrm{~K} \mathrm{[1].}$

Experimental Results and Discussions. The correlation signal measured as a function of the delay time is usually expressed by an exponentially decaying curve, from which we might estimate the decay time of photo-excited hot electrons. Alternatively we can trace the signal intensity at a given delay time as a function of the photon energy or

\footnotetext{
$\left.{ }^{1}\right)$ Present address: Toshiba Corporation, Kawasaki 210, Japan.

2) Present address: Denso Corporation, Showa-cho, Kariya 488, Japan.
} 


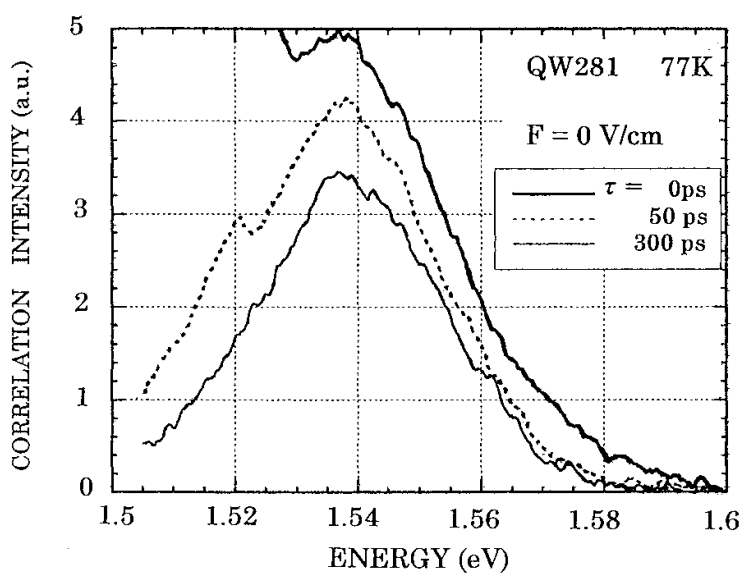

Fig. 1. Correlation signal intensity (always in arb. units) as a function of energy

the kinetic energy of electrons/holes. These curves reflect the temporal distribution of hot carriers in QW. Fig. 1 shows such traces. Obviously, the signal intensity is reduced, in the whole energy region, with the increase of the delay time (normal correlation), reflecting the decrease of carrier density at corresponding energies. By applying a high electric field along the wire axis to accelerate electrons, however, the decay curve exhibits anti-correlation behavior, i.e., the signal intensity increases with the delay time. Fig. 2 shows a typical result. In this case we get anti-correlation behavior at energies between 1.530 and $1.585 \mathrm{eV}$. The signal is negative at 1.552 to $1.585 \mathrm{eV}$. The results suggest that application of an external electric field makes a temporal accumulation (increase) of carriers at energies of 1.530 to $1.585 \mathrm{eV}$. The negative sign or strong accumulation is achieved at energies just below $1.585 \mathrm{eV}$, which sustained up to $300 \mathrm{ps}$. By analyzing the PL spectra at $77 \mathrm{~K}$, we found that the bottom of the $\mathrm{QW}$ is at $1.538 \mathrm{eV}$. From the S-dH measurements at $4.2 \mathrm{~K}$, the Fermi energy is estimated to be $10 \mathrm{meV}$ or at $1.548 \mathrm{eV}$. Therefore the strong accumulation is obtained if the kinetic energy of electrons measured from the Fermi energy is less than $37 \mathrm{meV}$, which is nearly equal to the LO phonon energy of GaAs $36 \mathrm{meV}$. These facts suggest that the accumulation of carriers due to the heating is effective only in the region where carrier cooling is dominated

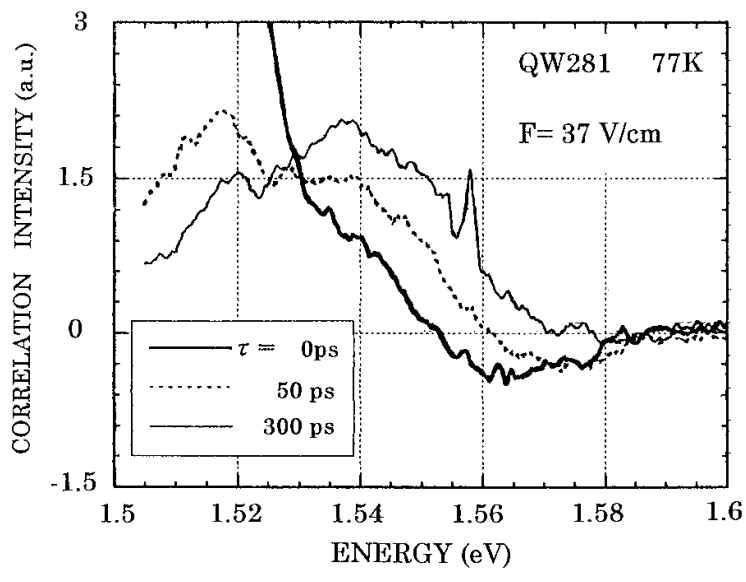

Fig. 2. Correlation signal intensity as a function of energy 
by the LA phonon emission process. This observation is in agreement with the theoretical prediction by Yamada and Sone [3], that carriers accelerated by an external electric field in a one-dimensional structure will accumulate at energies below the LO phonon emission threshold.

The results described above were obtained keeping the excitation intensity as low as possible (excited carrier density is estimated to be less than $10^{4} \mathrm{~cm}^{-1}$ ) so that the injected carriers might not modify the potential distribution within the wire. But the behavior of the correlation signal intensity was strongly dependent on the excitation intensity. If the excitation intensity was increased by one order of magnitude, i.e., at $10^{5} \mathrm{~cm}^{-1}$, which is still much less than the equilibrium electron density, the region of the negative sign was shrinked and shifted to high energies as shown in Fig. 3. Further increase of the excitation intensity results in total disappearance of the anti-correlation behavior under electric fields. This suggests simply that the electric field effect is smeared out by the high carrier density.

In Fig. 3, it is obvious that the negative sign is achieved even at energies above the LO phonon emission threshold. The relaxation of high energy electrons just after the injection into the QW with sufficiently high energies is due mainly to emission of LO phonons, while cooling rate for electrons the kinetic energy of which is less than $36 \mathrm{meV}$ is due to the emission of LA phonons and might be much smaller than those at above $36 \mathrm{meV}$. So, we may expect temporal accumulation of carriers just above the LO phonon energy emission threshold due to the rapid relaxation from the high energy region [4], in agreement with the observation. If this is the case, the negative sign should be observed after the completion of the capture and initial rapid decay of photo-excited hot electrons. Actually, at $\tau=0$, the negative sign is not so significant, but it becomes more prominent after several tens of picosecond as expected. Incidentally, we might always expect some sort of carrier accumulation above the LO phonon threshold irrespective to the excitation intensity. But, experimentally, we do not find clear evidence at low excitation intensities. This is attributed to the fact that the average electron temperature achieved by the photo-excitation is not high enough for the high energy tail in the PL spectra to be enhanced, which in turn would assist the increase of the carrier density at high energies.

Bockelmann and Bastard [5] studied the electron-LA phonon interaction in a onedimensional structure as a function of the wire width. According to their results, the

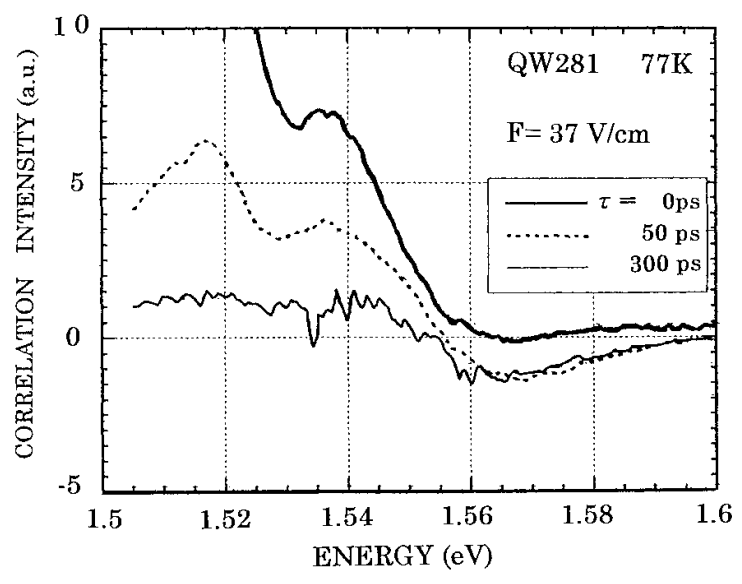

Fig. 3. Correlation signal intensity as a function of energy. High excitation, $F=$ $37 \mathrm{~V} / \mathrm{cm}$ 
emission rate is an oscillatory function of the wire width. But we might expect that the emission rate in a wire of $133 \mathrm{~nm}$ wide is reduced to less than a half of those in 2DEG. Experimentally, the negative sign is achieved in samples the geometrical width of which is less than $400 \mathrm{~nm}$ (effective width is around $270 \mathrm{~nm}$ ), in agreement with the theoretical prediction [5]. Similar measurements were performed for 2DEG samples, but we could not find any evidence of the negative sign at energies around the LO phonon emission threshold. Therefore, the accumulation of carriers around the LO phonon emission threshold is thought to be a specific nature in Q1D samples. According to the theoretical results due to Yamada and Sone [3], the occurrence of the accumulation under the LO phonon threshold will be observed if the LA phonon scattering rate is negligibly low. The accumulation of carriers above the LO phonon threshold, on the other hand, suggests that the LA phonon scattering rate is extremely lower than the LO phonon scattering rate. Therefore we might conclude that the LA phonon scattering rate must be much reduced in the Q1D structure as compared to those in 2DEG.

Summary. In summary, with the PL intensity correlation method, the cooling of photo-excited hot electrons in a quasi-one-dimensional structure has been studied, competing the heating effect due to the external electric field. Accumulation of carriers around the LO phonon emission threshold is attributed to the low LA phonon emission rate as compared to the high $\mathrm{LO}$ phonon emission rate.

Acknowledgements. This work is partly supported by the Grant in Aid from the Ministry of Education, Science, Sports and Culture of Japan. The excellent work of the SEM measurement of the wire widths by Mr. S. Tamura is acknowledged.

\section{References}

[1] D. von der Linde, J. Kuhl, and E. Rosengart, J. Lum. 24/25, 675 (1981).

[2] S. Niwa, T. Suzuki, N. Sawaki et al., Jpn. J. Appl. Phys. 34, 4515 (1995); 36, 3675 (1997).

[3] T. Yamada and J. Sone, Phys. Rev. B 40, 6265 (1989).

[4] Y. Awano, K. Tomizawa, and N. Hashizume, IEEE Trans. Electron Devices 31, 448 (1984).

[5] U. Bockelmann and G. Bastard, Phys. Rev. B 42, 8947 (1990). 